\title{
ERRATUM
}

\section{CONFORMAL FIELD THEORIES ON SURFACES WITH BOUNDARIES AND CROSSCAPS}

\author{
[MOD. PHY. LETT. A, Vol. 4, No. 2 (1989) 161 - 168] \\ T. ONOGI and N. ISHIBASHI
}

\author{
Please note the following typographical error on p. 163: Equation (6) should read$$
U L_{n} U^{\dagger}=\tilde{L}_{-n}
$$$$
V L_{n} V^{\dagger}=(-)^{n} \tilde{L}_{-n}
$$ 